\title{
Análisis clínico y citogenético en 257 casos de síndrome de Down
}

\author{
Carmen Astete A. ${ }^{1}$; Ronald Youlton R. ${ }^{1}$; Silvia Castillo T. ${ }^{1}$ \\ Cecilia Be R. ${ }^{2}$; Vera Daher N. ${ }^{1}$
}

Clinical and cytogenetic aspects in 257 cases of Down's syndrome

\begin{abstract}
Among 257 patients with clinical diagnosis of Down's 5yndrome, 56.4\% of male gender, in whom cytogenetic studies were performed, $14(5,4 \%)$ had normal karyotypes and $243(94.6 \%)$ had 21 trisomv. Of these last. $225(92.6 \%$ ) had free 21 trisomy, $10(4.1 \%$ ) showed mosaics. 8 (3.3\%) had translocations. Average maternal age of this sample was significantly higher than that of patients attending the same maternity wards 132.14 vs. 24.85 yearst and $41 \%$ of Down syndrome's patients carne from mothers aged 36 years or more, even though only $9.7 \%$ of this country's deliver ies proceed from women of that age group. As to seasonal ocurrence, the proportions of births which happened in summer almost doubled that of winter $\{33.1 \% \mathrm{vs} .16 .9 \%$. The frequency of characteristic clinical signs of Down's syndrome was somewhat different than that described for patients from some other countries, for instance: epicanthus, short rack and widening of the space between 1 st. and 2 nd. toe were more frequent, while Brushfield's spots, depressed occiput, dental anomalies, heart malformations and fisured tonge seemad less frequent. The importance of cytogenetic studies for diagnosis and genetic counseling is stressed.

(Key words: Down's syndrome, 21 trisomy, mosaicism, translocations, cy togenetics, genetic counseling).
\end{abstract}

El síndrome de Down fue la primera aberración cromosómica descrita en el hombre y es considerado el factor etjológico más común de retardo mental ${ }^{1}$.

La incidencia del sindrome de Down en Chile, según datos del Estudio Colaborativo Latinoamericano de Malformaciones Congénitas (ECLAMC) entre 1982 y 1986 , es de 16,2 ! 10.000 nacidos vivos (1/616 nacidos vivos) ${ }^{2}$.

En el servicio de genética del Hospital Clínico de la Universidad de Chile, entre 1981 y junio de 1990 , se realizó estudio citogenético a 1.638 pacientes; 243 de éstos $(14,8 \%)$ correspondieron a cuadros de trisomía 21 .

En el presente estudio se pretende caracterizar esta población con sindrome de Down, compararla con lo descrito en países de diferente etnicidad y buscar factores determinantes o relacionados con la génesis del desequilibrio cromosómico en los casos de trisomía 21 libre.

1. Servicio de Genética. Departamento de Medicina. Hospital Clínico Universidad de Chile.

2. Laboratorio de Genética. Clínica Las Condes.

\section{Material y Método}

Del registro de cariotipos del servicio de genética del Hospital Clínico de la Universidad de Chile se seleccionason todos los pacientes en que se realizó el examen, cuyo diagnóstico clínico đe referencia habiá sido síndrome de Dows $y, o$, aquellos cuyo cariograma dio como resultado una trisomia $2 \mathrm{k}$, ya fuera como trisomía 21 libre, mosaico o translocación.

Existe una ficha para sindrome de Down, en la que consta la identificación del paciente, datos del embarazo y del periodo de reciến nacido, una pequeña genealogía y un listado de los signos clínicos frecuentes en el síndtome. En algunos casos también se dispuso de las fichas clínicas del hospital, ks que se utilizaron para completar y corroborar algunos datos. Duscientos cincuenta y siete pacientes con diagnóstico clínico de síndrome de Down habían sido estudiados genéticamente y 156 de ellos tenian ficha de síndrome de Down.

Se compararon las frecuencias de trisomía 21 libre, translocación y mosaico en nuestro estudio con las frecuencias dadas por Giraud y $\mathrm{Mattei}^{3}$ y la frecuencia según sexo y estación de] año en que nacieron los pacientes, con las de la población control del hospital José Joaquín Aguirre 4 . Las comparaciones se realizaron mediante prueba de $\chi^{2}$. Se calculó la diferencia de tas medias para la edad entre las madres de los pacientes y la población de madres usuarias de la matemidad del aludido hospital, mediante prueba de $t$ para diferencias de las medias. 


\section{Resultados}

De los 257 pacientes con diagnóstico clinjco de sindrome de Down, 243 tenian cariotipo alterado $(94,6 \%)$ y 14 cariotipo normal $(5,4 \%)$. En estos últimos fue posible descartar clínicamente el diagnóstico a pesar de que presentaban algunas caracteristicas del súndrome. Sin embargo, el estudio citogenético fue igualmente realizado, ya que la duda habia sido planteada a los padres con anterioridad.

$\mathrm{La}$ frecuencia de las distintas alteraciones cromosomicas encontradas y la comparación con lo descrito por Giraud y Mattei en 4.760 $\operatorname{casos}^{3}$ se muestran en la tabla $\mathbf{l}$. No se encontraron diferencias significativas entre ambos estudios.

De las 225 trisomias 21 libre, 127 correspondían a pacientes de sexo masculino $(56,4 \%)$, distribución que no mostró diferencias significativas con la población de nacidos vivos en la

Tabla 1

Cariotipo en niños con sindrome de Daw'n

\begin{tabular}{|c|c|c|c|}
\hline \multirow[t]{2}{*}{ Cariotipo } & \multicolumn{2}{|c|}{ Hospital Clinico } & \multirow{2}{*}{$\begin{array}{c}\text { Giraud } y \\
\text { Mattei } \\
\%\end{array}$} \\
\hline & $n$ & $\%$ & \\
\hline Trisomia 21 libre & 225 & 92,6 & 92.5 \\
\hline Transloraciones & 8 & 3,3 & 4,8 \\
\hline $\begin{array}{l}\text { Dq } 21 \mathrm{q} \\
\mathrm{Gq} 21 \mathrm{q}\end{array}$ & $\begin{array}{l}(3,00) \\
(5,00)\end{array}$ & $\begin{array}{l}(1,24) \\
(2,06)\end{array}$ & $\begin{array}{l}(2,60) \\
(1,96)\end{array}$ \\
\hline Otros & & & $(0,24)$ \\
\hline Mosajcos & 10 & 4,1 & 2,7 \\
\hline
\end{tabular}

maternidad del hospital José Joaquín Aguirre entre 1982 y $1986^{4}\left(\chi^{2}=2,74 \mathrm{p}<0,05\right.$, $\mathrm{p}=0,098)$.

La edad materna promedio, calculada para 143 casos de trisomia 21 libre, fue 32,17 años (DE 7,98), significativamente mayor que la edad materna promedio en la maternidad del hospital José Joaquín Aguirre entre 1969 y 1979 , que fue de $24,85 \operatorname{anos}^{5}(t=10,61 \mathrm{p}<0,001)$. Los promedios de edad materna en los casos de mosaico y de translocación fueron menores (31 años y 29,3 años, respectivamente) pero no se analizaron debido al tamaño de la muestra.

El porcentaje de nifos con sindrome de Down que habia en cada grupo etario materno se comparó con el porcentaje de natalidad que le corresponde a cada gnupo según el total de nacidos vivos del país ${ }^{6}$ (tabla 2): $41,26 \%$ de los niños con síndrome de Down eran hijos de madres de 36 años y más, en tanto que sólo 9,7\% del total de nacidos vivos correspondieron a este grupo.

La proporción de niños que nacieron en las distintas estaciones del año, considerando verano a los meses de dicienbre, enero y febrero; otoño los meses de marzo, abril y nuayo; invierno a junjo, julio y agosto, y prinavera a septiembre, octubre y noviembre, fue la siguiente: $23,5 \%$ de los casos nacieron en otono; $26,5 \%$ en primavera; $33,1 \%$ en verano y $16,9 \%$ en inviemo. Esto se comparó con lo observado en la maternidad del hospital José Joaquin Aguirre entre los años 1969 y $1988^{5}$. La variación estacional observada está en el limite de la significancia $\chi^{2}=7,74 p=0,052$.

Ochenta y cuatro por ciento de los exámenes citogenéticos fueron realizados antes que los

Tabia 2

Proporciones de niños nacidos con sindrome de Dow'n $y$ del total de ndcidos vivas para diferentes categorías de edad materna

\begin{tabular}{|c|c|c|c|c|}
\hline \multirow{2}{*}{$\begin{array}{l}\text { Rango de edad } \\
\text { materna } \\
\text { años }\end{array}$} & \multicolumn{2}{|c|}{ Síndrome de Down } & & \multirow{2}{*}{$\begin{array}{c}\text { Total nacidos vivos } \\
\%\end{array}$} \\
\hline & $\mathbf{n}$ & $\%$ & & \\
\hline$<20$ & 12 & 8.39 & & 13,5 \\
\hline $21-25$ & 26 & 18,18 & & 31,1 \\
\hline $26-30$ & 21 & 14.69 & & 28,6 \\
\hline $31-35$ & 25 & 17,48 & & 17,1 \\
\hline $36-40$ & 35 & 24,48 & & 7,7 \\
\hline$>41$ & 27 & 16,78 & $41,26 \%$ & $2.0\}^{9,7 \%}$ \\
\hline
\end{tabular}


pacientes tuvieran 12 meses y $24,7 \%$ eran menores de un mes en el momento del estudio.

Las frecuencias de presentación de los 23 signos clínicos más caracteristicos del síndrome de Down, en comparación con las descritas por O.J. Miller (tabla 3$)^{7}$, resultaron llamativamente mayores para epicantus, puente nasal bajo, cuello corto y separación entre primero y segundo ortejos en nuestra serie; en cambio las manchas de Brushfield, el occipuccio plano, anomalías dentales, lengua fisurada y cardiopatia congénita fueron menos frecuentes que en la serie mencionada.

\section{Comentario}

El sindrome de Down es considerado el factor etiológico más común de retardo mental. En Chile, con una incidencia de $16,2 / 10.000$ nacidos vivos ${ }^{2}$, nacerían aproximadamente 450 niños con este trastorno al año. Las cifras actuales de incidencia en los paises desarrollados son de 1/1.000 nacidos vivos?. Esta importante diferencia se atribuye, por una parte, a la mejor información que tiene la población sobre los factores de riesgo (edad materna), lo que influye sobre la planificación familiar $y$, por otra, a que poblaciones de mayor riesgo pudiesen tener mayor acceso a los métodos de diagnóstico antenatal y subsecuentemente a interrupción del embarazo en los casos en que se detectan altera. ciones cromosómicas.

La edad promedio de las madres de niños con sindrome de Down es superior a la edad materna promedjo en la maternidad del hospital José Joaquin Aguirre. Este es el factor más ampliamente reconocido cono relacionado con el mayor riesgo de tener hijos con trisomia $21^{8-10}$. Desincentivar los embarazos en mujeres mayores es una medida importante para la prevención, en este caso.

En busca de influencias ambientales no se encontraron diferencias estadísticamente significativas con respecto a la estacionalidad de los partos; sin embargo, es notable que la proporción de nacidos con síndrome de Down es casi el doble en el verano que en el inverno. Previa. mente se han descrito variaciones estacionales, pero las diversas publicaciones no coinciden en los meses o en la estación del año, de acuerdo al hemisferio".

Tabla 3

Frecuencia relativa y comparada ${ }^{7}$ de las principales manifestaciones clínicas del sindrome de Down

\begin{tabular}{|c|c|c|c|}
\hline & Signos clínicos & Hospital J. J. Aguirte & $\begin{array}{l}\text { Miller } \\
\%\end{array}$ \\
\hline 1 & Retraso psicomotor & $76 * *$ & 100 \\
\hline 2 & Hipotonjá & 86 & 77 \\
\hline 3 & Hiperextensibilidad & 76 & $47-77$ \\
\hline 4 & Rasgos faciales planos & 85 & 90 \\
\hline 5 & Fisuras palpebrales oblicuas & 87 & 80 \\
\hline 6 & Epicantus & 85 & $40 *$ \\
\hline 7 & Manchas de Brushfield & $18 *$ & 50 \\
\hline 8 & Anomalías de las orejas & 48 & 50 \\
\hline 9 & Puente nasal bajo & 83 & 60 \\
\hline 10 & Paladar estrecho ojival & 74 & 70 \\
\hline 11 & Braquicefalia & 69 & 75 \\
\hline 12 & Occipucio aplanado & $46^{*}$ & 78 \\
\hline 13 & Anomalías dentales & $2 *$ & 65 \\
\hline 14 & Lengun fisurada & $10 *$ & 50 \\
\hline 15 & Cuello corto & 77 & $45 \neq$ \\
\hline 16 & Cardiopat is congénita & $26 *$ & $40-60$ \\
\hline 17 & Extremidades cortas & 64 & 70 \\
\hline 18 & Manos anchas & 73 & 70 \\
\hline 19 & Clinodactilia $5^{\circ}$ dedo & 67 & 50 \\
\hline 20 & Pliegue único $50^{\circ}$ dedo & 32 & 20 \\
\hline 21 & Surco palmar transverso & 53 & 48 \\
\hline 22 & Separación 10 y $2^{\circ}$ ortejos & 85 & $45 *$ \\
\hline 23 & Surco plantar & 61 & $28 *$ \\
\hline
\end{tabular}

* Frecuencia significativamente menor que la otra serie.

** 24\% fue examinado antes del mes de edad. 
El cuadro clínico del síndrome de Down está muy bien caracterizado ${ }^{7}$ y la frecuencia de presentación de los distintos signos es bastante alta, lo que permite al examinador hacer el djagnóstico en forma precisa la mayoria de las veces. La menor frecuencia de presentación de las anomalias dentales, lengua físurada y cardiopatía congénita que observamos en nuestra población se debe, probablemente, a que el examen clínico se realizó en muchos casos a una edad en que aún no están presente estas alteraciones. El epicantus y las manchas de Brushfield se presentaron con distinta frecuencia, posiblemente por diferentes influencias étricas, como ocurre probablemente tambjén con el cuello corto, el occipucio aplanado y los dismorfias de los pies.

Conviene destacar la importancia que tiene hacer el estudio citogenético en todos los casos, sin excepción, en que se propone el diagnóstico de síndrome de Down, por ser esta la única forma de confimarlo $y$ deteminar si se trata o no de una translocación, puesto que el riesgo de recurrencia es muy diferente cuando la alteración es heredada de uno de los padres. También es de mucha importancia el estudio cjtogenético en el caso de tener dudas sobre el diagnóstico, como queda ilustrado por los casos que en este estudio fueron referidos como sindrome de Down, pero cuyo cariotipo fue normal. Si bien es cierto que en todos ellos fue posible descartar clínjcamente el diagnóstico, el examen citogenético es el único elemento objetivo una vez planteada la duda.

\section{Resumen}

Se analiza una población de 257 pacientes, $56,4 \%$ varones, con diagnóstico clínico de sindrome de Down, a los que se les realizó estudio citogenético. Catorce $(5,4 \%)$ tenían cariotipo nonnal y $243(94,6 \%)$ trisomía 21 . De estos últimos, $225(92,6 \%)$ correspondian a trisomía 21 libre, $10(4,1 \%)$ a mosaicos, 8 $(3,3 \%)$ a translocaciones. El promedio de edad materna fue significativamente mayor que el de las madres atendidas en la maternidad del hospital José J. Aguirre (32,14 vs. 24,85 años) y 41,26 por ciento de los pacientes con sindrome de Down era hijo de madres de 36 años y más, en circunstancias que al mismo grupo etarjo corresponde sólo $9,7 \%$ de los partos que ocurren en el país. Los nacimientos según estación del año fueron casi el doble en verano $(33,1 \%)$ que en inviemo $(16,9 \%)$. La frecuencia de presentación de algunos signos clínicos característicos del síndrome de Down como epicanto, puente nasal bajo, separación entre primero y segundo ortejos fue mayor que lo descrito en otros países, mientras que otras como manchas de Brushfield, occipucio plano, malformaciones dentales y cardiovasculares fue menor, lo que, en parte, es atribuible a la edad en que se realizó el examen clínico. Se destaca la importancia del estudio citogenético para dar un consejo genético apropiado.

(Palabras clave: síndrome de Down, trisomía 21, mosaico, translocación, citogenética, consejo genético.)

\section{Referencias}

1. Moser, H.V.; Ramey, C.T. and Leanard, C.O.: Mental Retardation. En: Emery, A.: Rimoin, D.; Foreword by V.A. Mc Kusick Principles and Practice of Medical Genetics. London: Churchill Livingstone; Medical Division Group Limited. $1983 ; 353-366$.

2. Nazer, J.: Castillo, S.: Cifuentes, L.: Ruiz, G,; Pizarro, M.T.: Parada, L.: Frecuencia de malformaciones congénitas en Chile en et perjodo 19691986. Resultados de un estudio colaborativo latinoamericano. Rev Med Chile 1989; 117; 219. 227.

3. Glraud, F, Mattei, J.F.: Aspects epidemiologiques de la trisomie 21. J Génét Human 1975; 23: 1-30.

4. Nazer, J.; Cifuentes, l.; Catalion, J, y cols.: Incidencia de malformaciones congénitas en mortinatos. Rev Chil Pediatr 1989; 6: 208-217.

5. Cifuentes, L.: Nazer, J, Catalón, J.: Malformaciones congénitas: Un modelo predictivo basado en factores de riesgo. Rev Med Chile 1989; 117 : 611-617.

6. Anonimo: Anuario de Demografía 1988. República de Chile; Ministerio de Economía, Fomento y Reconstrucción; Instituto Nacional de Esta. dísticas (JNE): Ministerio de Salud; Servicio de Registro Civil e Jdentificación. Imprenta INE, 1990.

7. Miller, OJ.: Chromosome twenty one Syndrome en Bergsma, D. Birth Defects Compendium $N$. York: Edit. Alan Liss Inc. 1979; 215-216.

B. Scatbrough, P.; Finley, W.: Finley, S.: A Review of trisomies 21,18 and 13 . The Alabama Journal of Medical Sciences. 1982; 19: 174-186.

9. Castilia, E; Orioli Panerias, L.; Madeiros Simoes. M.A.: Estudio de Factores de Rjesgo en el Síndrome de Down. Pediatría (Stgo.). 1980; 23: 89104.

10. Nozer, J.: Rizzardini, M.; Diaz, G.: Walton, $R$ : Malformaciones congénitas. VIl. Síndrome de Down. Pediatria (Stgo.). 1980; 23: 82-88.

11. Litienfeld, A.M.: Epidemiology of Mongolism. Maryland: Johns Hopkins. $1969 ; 56-58$. 